\title{
Complex structures in media displaying electromagnetically induced transparency: pattern multistability and competition
}

\author{
M. Eslami ${ }^{1}$, R. Kheradmand ${ }^{1}$, D. McArthur ${ }^{2}$, and G.-L. Oppo ${ }^{2 *}$ \\ ${ }^{1}$ Photonics Group, Research Institute for Applied Physics and Astronomy, University of Tabriz, Tabriz, Iran and \\ ${ }^{2}$ SUPA and Department of Physics, University of Strathclyde, Glasgow G4 ONG, Scotland, UK
}

\begin{abstract}
Spatially periodic and localized structures in the transverse plane of a medium displaying electromagnetically induced transparency in an optical cavity and under the action of two pumps, are investigated. The system supports a multitude of different complex spatial structures depending on the chosen initial condition. We explore regimes of multistable patterns, filaments, stable defects, scrolling structures, nested patterns, fronts and the spontaneous occurrence of multiple cavity solitons. To simulate realistic conditions of operation, we replace periodic boundary conditions with pumps of finite size. Many of the multistable features are recovered apart from the scrolling of patterns with defects.
\end{abstract}

PACS numbers: 42.50.Lc, 42.50.Dv, 42.65.Yj

\section{INTRODUCTION.}

Formation of regular and localized structures in spatially extended systems far from thermodynamical equilibrium has been the subject of a vast number of researches in the past two decades $[1-5]$. For optical systems spatio-temporal phenomena arise in the structure of the electromagnetic field in the plane orthogonal to the direction of propagation as a result of the nonlinear response of the materials to intense laser beams and the spatial coupling provided by diffraction. Diffraction in the paraxial approximation is described by a transverse Laplacian operator. Particularly interesting is the case of nonlinear materials contained in optical cavities under the action of external pumps. In the mean field approximation, such systems are described by complex partial differential equations $[3,5,6]$ with two spatial dimensions plus time. Stationary solutions of partial differential equations can be seen as single points in an infinite dimensional phase space. The identification of families of coherent structures (or modes) allows, however, to reduce the infinite degrees of freedom to a finite number of relevant variables and move within a finitedimensional sub-space. When a bifurcation occurs, the associated unstable trajectories move away from the original stationary point but remain typically confined to a lower dimensional sub-space of the fully available volume. This sub-space is attracting in the sense that trajectories starting outside such space will converge to it, so that the degrees of freedom outside the attractors are effectively irrelevant $[7,8]$. It is for this reason that it is possible to describe several pattern-formation problems near thresholds of instability with a limited number of families of solutions (modes). After an instability has produced a growing disturbance from one of the stationary modes, intrinsic nonlinearities move the system toward a new

*Electronic address: g.1.oppo@strath.ac.uk state. In some cases local disturbances grow to finite amplitudes and the new state resembles a deformation of the original structure with stable defects. In other cases, the new structure, that can be a stationary state or a dynamical regime (including spatio-temporal chaos), looks nothing like the linearly unstable deformation from which we have started [9]. The system may evolve in entirely new directions as determined by the nonlinear dynamics, multistable solutions and the initial condition. The importance of initial conditions for the asymptotic behaviour of the system is well-known from the basic concepts of nonlinear dynamics and complex systems. In our case sensitivity to initial conditions means that each point in phase space may be very close to other points with significantly different future evolutions. Thus, an arbitrarily small perturbation of the current trajectory may end in one of the many stable solutions. Once the evolution has stabilized, memory of the initial condition can affect the sequence of bifurcations of the asymptotic structure observed when changing a control parameter.

The complex spatial structures forming in nonlinear cavities displaying electromagnetically induced transparency (EIT) [10] show generalized multistability of the kind described above. In particular, it will be shown that different spatially periodic structures (optical patterns) are obtained for different initial conditions but the same parameter values. Local perturbations lead to stable patterns with defects. When changing a control parameter the sequence of observed structures including distorted, oscillating and scrolling (DOS) solutions displays memory of the chosen initial condition. These effects survive the presence of optical pumps of finite size. The simultaneous presence of a multitude of extended and localized spatial structures, defects, disorder, front instabilities and filamentation provides EIT media in optical cavities with unique flexibility and control of operation with possible applications in optical processing of information, novel memory functions and self-organized compensation of diffraction.

The paper is organized in the following sequence: the 
(a)

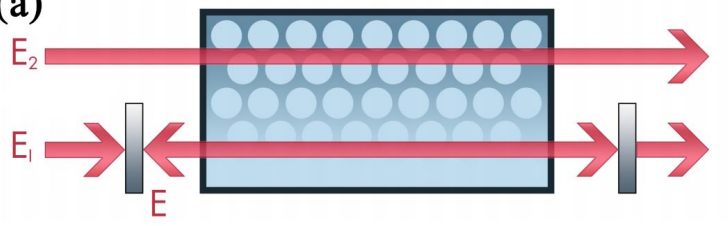

(b)

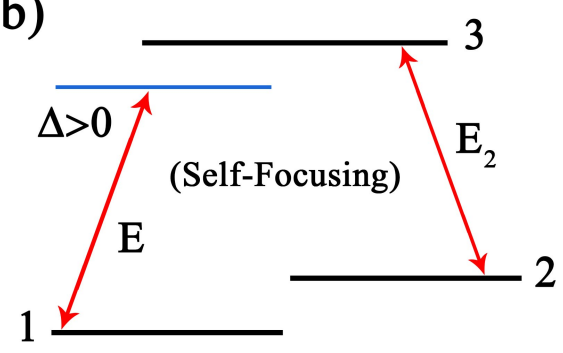

FIG. 1: (Color online) (a) The cavity configuration with the three-level atomic medium and the holding $E_{I}$ and coupling $E_{2}$ beams. (b) The $\Lambda$ atomic scheme with two ground states $|1>| 2>$, and a single excited level $\mid 3>$.

model and associated equations are discussed in section 2 along with the stationary solutions and their stability analysis. Section 3 describes the variety of solutions obtained by using different initial conditions. We then describe the spontaneous appearance of a cavity soliton gas in Section 4 while the effects of finite pump are presented in Section 5. Conclusions and topics for future research are discussed in Section 6.

\section{THE MODEL.}

The system of interest is a Fabry-Perot type cavity filled with 3-level atomic vapour (for example Rb atoms) in a $\Lambda$ configuration and under the action of two optical pumps. The schematic representation of the cavity and the configuration of atomic vapour are shown in Fig. 1. The injected field $E_{I}$ (holding beam) is detuned by $\Delta$ from resonance of the atomic transition $|3>\rightarrow| 1\rangle$ while the coupling beam $E_{2}$ is kept at resonance with the transition $|3>\rightarrow| 2>$. The cavity mirrors resonate the field $E$ which is detuned form the injection $E_{I}$ by $\theta$. In the present model the field $E_{2}$ is not resonated in the cavity which is realistic if the atomic frequencies are well separated. Many EIT experiments, however, use the ground states of alkali atoms with a small frequency difference (few $\mathrm{GHz}$ ) between the two optical fields. In such cases, polarizing beam splitters can be used to introduce orthogonal polarizations so that the coupling beam $E_{2}$ is not oscillated in the cavity [11].

The mean field equation for a beam propagating in the $\Lambda$ medium inside the optical cavity of Fig. 1 is [10]:

$$
\partial_{t} E=E_{I}-(1+i \theta) E-2 i C \rho_{13}+i \nabla^{2} E
$$

where $E$ is the complex intra-cavity field, $E_{I}$ is the normalized amplitude of the pump field (considered to be a real function without loss of generality), $\theta$ is the detuning between the cavity resonance and the frequency of the injected pump beam, and $\rho_{13}$ is the off-diagonal density matrix element proportional to the field amplitude $E$ and the complex susceptibility $\chi$ via the relation

$$
\rho_{13}=\chi E=\frac{\Delta\left|E_{2}\right|^{2}\left(\left|E_{2}\right|^{2}+|E|^{2}-i \Delta\right)}{\left(\left|E_{2}\right|^{2}+|E|^{2}\right)^{3}} E .
$$

$C$ is the co-operative parameter directly proportional to the atomic density $n_{a}$ through

$$
2 C=\frac{n_{a} \mu^{2} k L}{2 \hbar \gamma \epsilon_{0} T},
$$

where $\mu$ is the atomic transition dipole moment, $k$ the wave number of the field, $L$ the length of the cavity, $\gamma$ the atomic linewidth, $\epsilon_{0}$ the permittivity of free space, and $T$ is the cavity mirror transmittivity. The diffraction term is given by the Laplacian operator in two transverse dimensions and time is normalized to the photon life time. Details of the derivation of the diffractive Maxwell-Bloch equation (1) for the case of a two-level medium are provided in $[6,12]$. Equation (1) is a generalization of the model introduced in [10] since in the evaluation of $\rho_{13}$ we used a less stringent condition of $|\Delta|^{2}<<\left|E_{2}\right|^{2}$ instead of $|\Delta|<<\left|E_{2}\right|^{2}$. The bistable nature of the light-atom interaction and the capability of the system for displaying EIT in the present model (1) are extended to a wider parameter space than that of [10] (see for example Fig. 2 where the complex susceptibility and the input-output curve are displayed in (a) and (b), respectively). Note that the detuning parameter $\Delta$ is here considered to be positive indicating the self-focusing regime. The homogeneous steady states are given by the implicit complex equation

$$
E_{I}=(1+i \theta) E_{s}+\frac{2 i C \Delta\left|E_{2}\right|^{2}\left(\left|E_{2}\right|^{2}+\left|E_{s}\right|^{2}-i \Delta\right)}{\left(\left|E_{2}\right|^{2}+\left|E_{s}\right|^{2}\right)^{3}} E_{s}
$$

where $E_{s}$ is the steady value of the intra-cavity complex field. The linear stability analysis of the homogeneous steady state solutions determines the Turing instability domain where they bifurcate to patterned structures. The characteristic equation for the critical wave-vector $K$ of the patterned structures is obtained from linear stability analysis calculations and is given by

$$
\begin{aligned}
K^{2}= & -\left[\theta+\frac{2 C \Delta\left|E_{2}\right|^{2}\left(\left|E_{2}\right|^{2}-\left|E_{s}\right|^{2}\right)}{\left(\left|E_{2}\right|^{2}+\left|E_{s}\right|^{2}\right)^{3}}\right] \\
& \pm\left\{\left[\frac{2 C \Delta\left|E_{2}\right|^{2}\left|E_{s}\right|^{2}}{\left(\left|E_{2}\right|^{2}+\left|E_{s}\right|^{2}\right)^{3}}\right]^{2}\left[4+\frac{9 \Delta^{2}}{\left(\left|E_{2}\right|^{2}+\left|E_{s}\right|^{2}\right)^{2}}\right]\right. \\
& \left.-\left[1+\frac{2 C \Delta^{2}\left|E_{2}\right|^{2}\left(\left|E_{2}\right|^{2}-2\left|E_{s}\right|^{2}\right)}{\left(\left|E_{2}\right|^{2}+\left|E_{s}\right|^{2}\right)^{4}}\right]^{2 / 2}\right\}^{1 / 2} .
\end{aligned}
$$



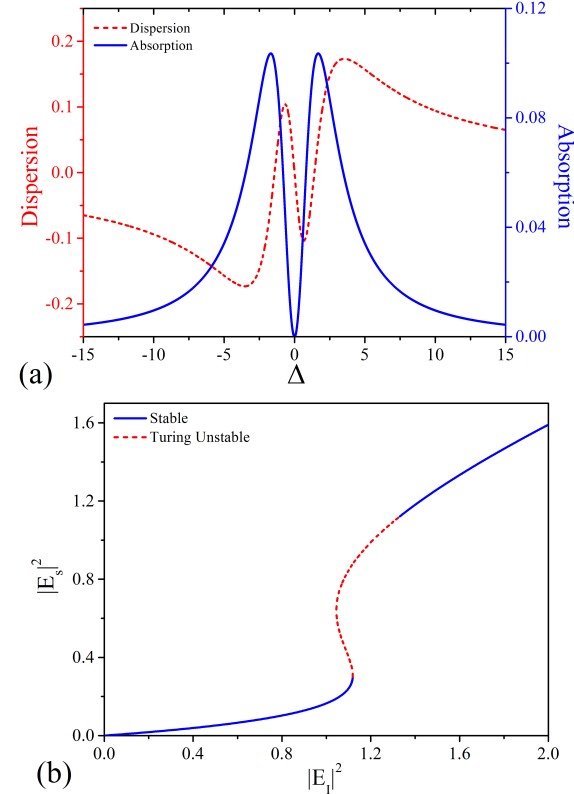

FIG. 2: (Color online) (a) The imaginary (solid line) and real (dashed line) parts of the complex susceptibility $\chi$ for $|E|^{2}=\left|E_{2}\right|^{2}=1$. (b) Bistability in the input and output intensities for $\Delta=0.2, \theta=-1,\left|E_{2}\right|^{2}=1$ and $2 C=20$.

For example, for the set of parameter values chosen in Fig. 2 (b), the dashed line indicates the region where the homogeneous solution leads to patterns while in Fig. 3 (a) the Turing bifurcation point occurs at $2 C=18.8$ for $I_{s}=\left|E_{s}\right|^{2}=1$. The range of unstable wave-vectors is displayed in Fig. 3 (b) where the entire region enclosed in the curves is Turing unstable. In Fig. 3 (b) we also display the line corresponding to the wave vector with maximum growth rate:

$$
K_{\max }^{2}=\frac{2 C \Delta\left|E_{2}\right|^{2}\left(\left|E_{s}\right|^{2}-\left|E_{2}\right|^{2}\right)-\theta\left(\left|E_{s}\right|^{2}+\left|E_{2}\right|^{2}\right)^{3}}{\left(\left|E_{2}\right|^{2}+\left|E_{s}\right|^{2}\right)^{3}} .
$$

It is obvious that for the case of $I_{s}=\left|E_{s}\right|^{2}=\left|E_{2}\right|^{2}$ one obtains a straight line at $K_{\max }=\sqrt{-\theta}$ not displayed in Fig. 3 (a).

Although the fundamental mechanism of spatial coupling in optics is diffraction instead of diffusion, the nature and character of the pattern forming instabilities displayed here are the same of those introduced by Alan Turing in 1952 [13] as discussed in [14].

\section{MULTISTABLE SPATIAL STRUCTURES}

We have numerically integrated Eq. (1) by using a split-step method where we separate the algebraic and Laplacian terms and solve the time derivative term by a Runge-Kutta algorithm and diffraction term by Fast Fourier Transforms. Simulation grids up to $256 \mathrm{x} 256$
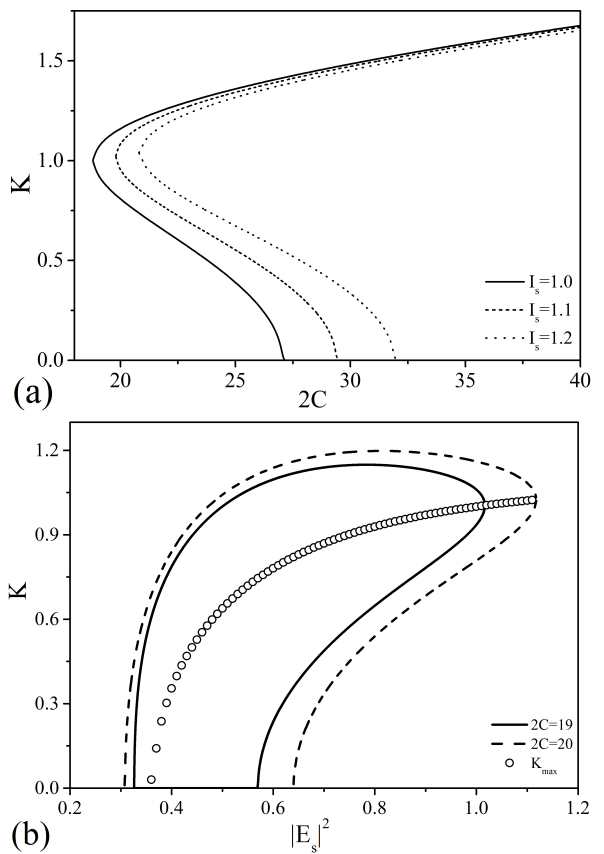

(b)

FIG. 3: Turing instability domains for pattern formation of wave-vector $K$ versus increasing $2 C$ (a) and versus increasing stationary intensities (b). Parameter values are $\Delta=0.2$, $\theta=-1,\left|E_{2}\right|^{2}=1$. In (a) $\left|E_{s}\right|^{2}=1$ (solid line), $\left|E_{s}\right|^{2}=1.1$ (dashed line) and $\left|E_{s}\right|^{2}=1.2$ (dotted line). In (b) $2 C=19$ (solid line) and $2 C=20$ (dashed line). The line traced by the small circles determines the wave-vector of largest growth for $2 C=20$.

points have been used while changing the control parameter $2 C$ that can be modified experimentally by increasing or decreasing the atomic density $n_{a}$. In what follows three regimes based on different initial values of the control parameter are discussed, each of them displaying separate characteristics for the observed sequence of solutions. Parameter values are kept fixed at $\Delta=0.2$, $\theta=-1,\left|E_{2}\right|^{2}=1$ and $\left|E_{s}\right|^{2}=1$ unless stated otherwise.

\section{A. Stable periodic patterns}

The bifurcation point of the homogeneous solution to patterns in Fig. 3 (a) is $2 C=18.8$. In the first scan the initial value of $2 C$ is selected below the bifurcation point. This leads to the sequence of stable patterns changing from honeycombs to rolls and from rolls to spontaneous cavity solitons towards the end of the instability interval. Stable honeycombs start at $2 C=18.8$ and end at 19.8, stable rolls start at $2 C=19.9$ and end at 21.61 , cavity solitons are observed between $2 C=21.62$ and 21.6265 . Examples of these patterns along with their far-field images are shown in Fig. 4 for $2 C=19.6$ (a)-(b), $2 C=21.0$ (c)-(d) and $2 C=21.62$ (e)-(f).

The sequence of the patterns and the associated inten- 


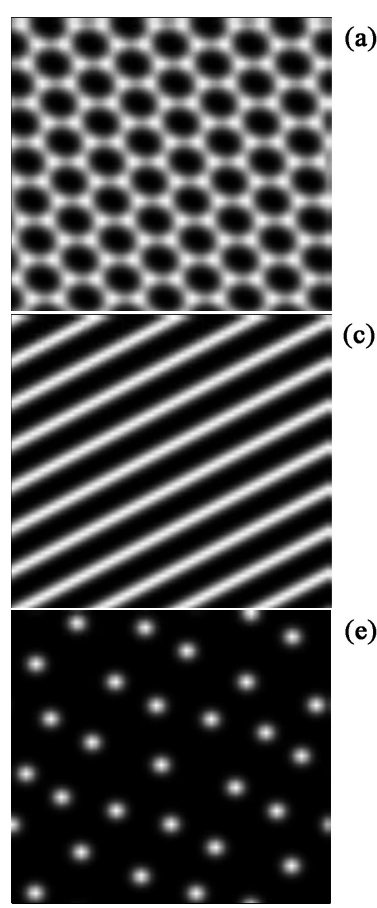

FIG. 4: (a) Stable honeycomb structures for $2 C=19.6$, (c) stable roll patterns for $2 C=21.0$, (e) cavity solitons for $2 C=21.62$ with (b), (d) and (f) their far-field images respectively. Note that in (b), (d) and (f) the central point has been removed to increase clarity of the images.

sities are shown in Fig. 5 versus the corresponding input intensity $\left|E_{I}\right|^{2}$.

\section{B. Distorted and scrolling structures}

We turn our attention to a starting point of the simulations just after the Turing instability point $(2 C=18.9$ for our selected parameter values). A graph showing the sequence of different transverse structures and their intensities versus changes of the control parameter $2 C$ is shown in Fig. 6. Structures with different characteristics from the regular patterns described above are observed from the very beginning of the scan. The black squares in Fig. 6 correspond to honeycomb patterns with stable defects that alter their spatial periodicity. For example in Fig. 7 we present a stable honeycomb structure, distorted by defects, and its far field distribution obtained by starting from random initial conditions. In general, defects appear when there are more than one attracting solution in the dynamics of a given system. If the initial condition puts the spatial configuration of the field somewhere between the attracting solutions, stable fronts and defects between the two patterns can form as described for example in chapter 7 of [1]. In the cases where the two attracting solutions are the same pattern but with different wave-vectors, formation of defects are expected

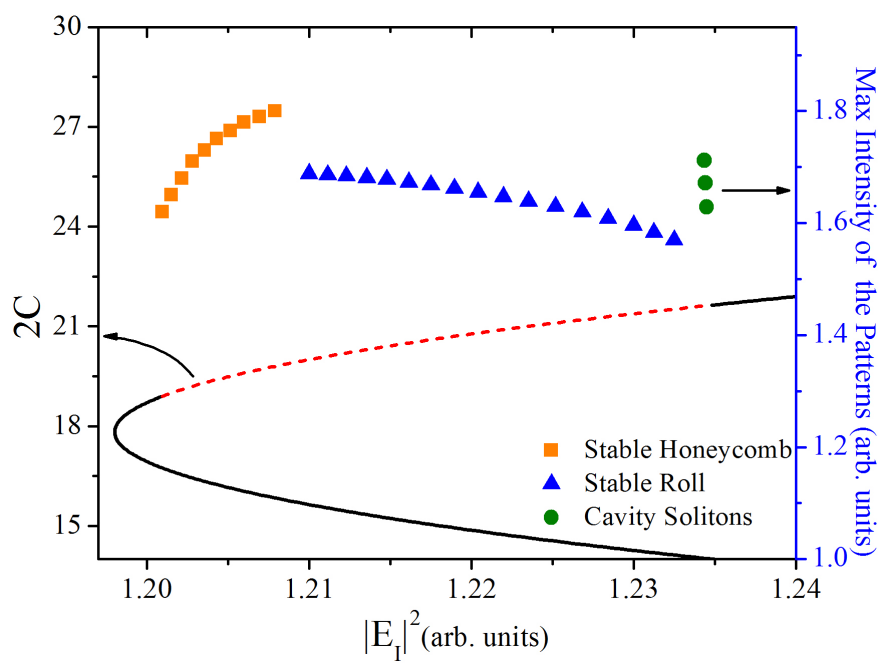

FIG. 5: (Color online) The instability domain of the $2 C$ parameter versus the input intensity (lower part and left axis) and the corresponding maximum intensities for different patterns (upper part and right axis).

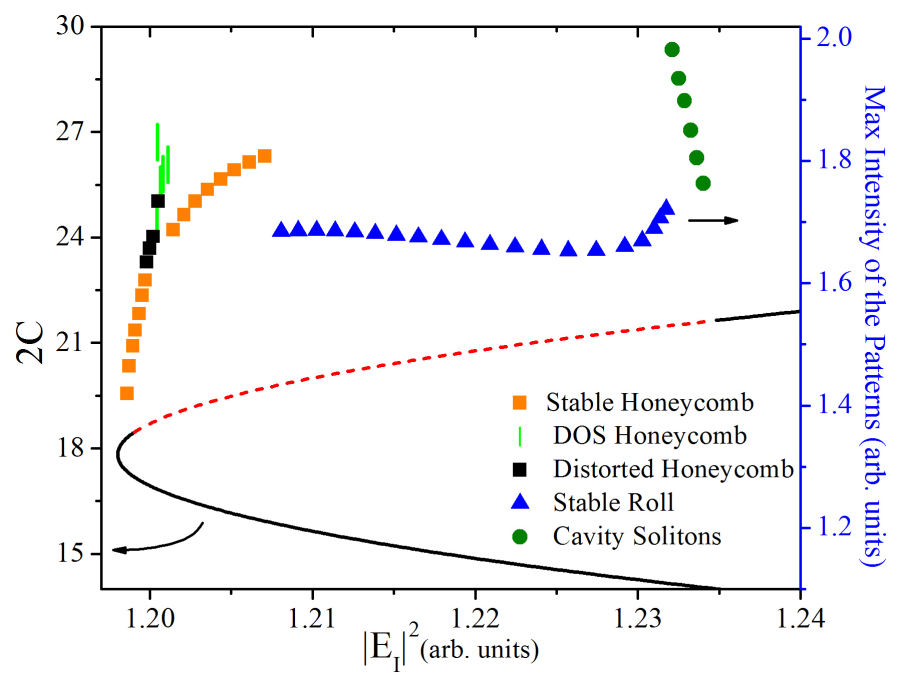

FIG. 6: (Color online) The instability domain of the $2 C$ parameter versus the input intensity (lower part and left axis) and the corresponding maximum intensities for different patterns (upper part and right axis) when starting at $2 C=18.90$. "DOS" stands for Distorted-Oscillating and Scrolling.

while for the case of two different patterns associated to the attracting solutions, nested patterns can form [15] as discussed in the next subsection.

At first transverse patterns with defects are stationary but then they unlock to form scrolling structures that move across the transverse space when increasing $2 C$. Scrolling structures have been studied in [16] where a constant velocity of scrolling has been observed in a variety of simulations of optical systems. In Fig. 8 (a) we show the frequency of the scrolling motion (dotted line) and the os- 


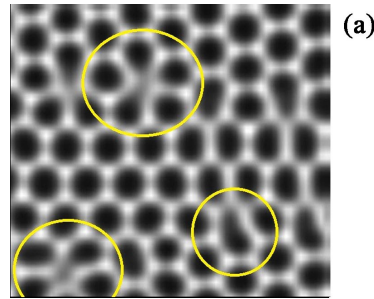

(b)

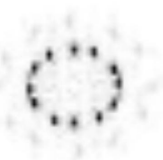

FIG. 7: (a) The distorted honeycomb pattern (left) and its far-field image (right) for $2 C=18.84$. The yellow circles identify the position of defects. Note that these patterns with defects maintain their spatial shape basically unchanged when turning into scrolling structures.
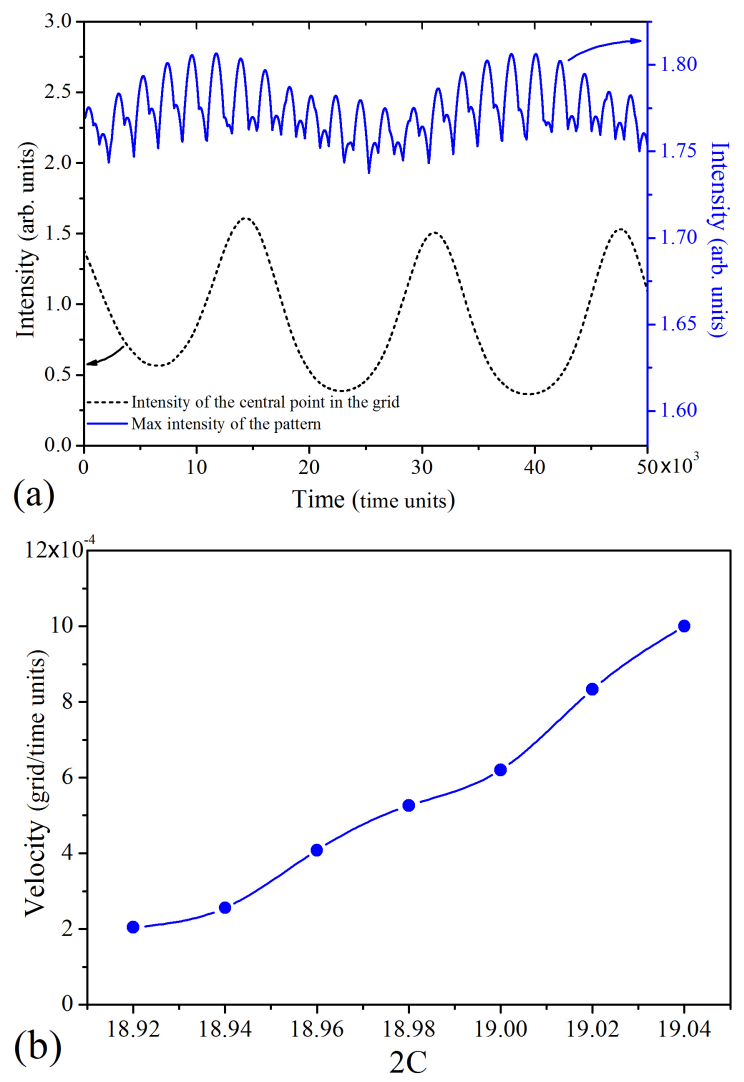

FIG. 8: (a) Oscillation of the maximum intensity of a scrolling pattern with defects (solid line) and in the central point of the structure (dotted line) for $2 C=19.0$. (b) Speed of the scrolling patterns versus $2 C$.

cillation of the maximum intensity (solid line) that has a frequency around 8 times larger than that of the scrolling motion. When increasing $2 C$ further, the scrolling speed increases (see Fig. 8 (b)) until locking takes place again. Two ranges of scrolling structures have been found in the simulations of Fig. 6 , from $2 C=18.88$ to 18.89 and from $2 C=18.92$ to 19.04 . When increasing further the control parameter $2 C$, regular honeycombs are retrieved although with different orientation and slightly different

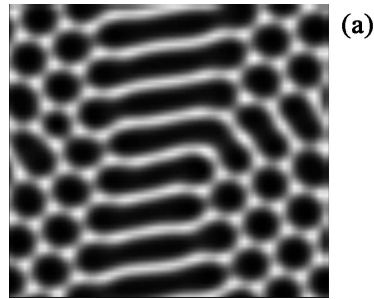

(b)

FIG. 9: Stable fronts separating roll patterns nested in a honeycomb structure (a) and their far-field image (b) for $2 C=19.6$.

wave-number than those described in III A. When these become unstable, roll structures are formed but with an intensity and a wave-vector widely different from those observed in III A. This is not surprising since the number of stable wave-vectors increases with $2 C$ as shown in Fig. 3 . When finally roll patterns lose stability, the regime of soliton gas has a stability range and intensity different from what has been observed above, thus confirming the sensitivity of the observed structures and bifurcations upon the initial conditions of the scan.

\section{Bistable patterns and wavy rolls}

Setting the initial value of the control parameter $2 C$ to 19.6 and scanning the Turing unstable interval up and down, further dependencies from the initial conditions emerge. We are now in a regime of bistable roll and honeycomb patterns. Stable fronts separating the two patterns can develop during the temporal evolution from random initial conditions. In the simulations presented here we find rolls surrounded by (nested in) honeycombs in the $2 C$ range of 19.55-19.75. Coexisting honeycomb and roll patterns in nonlinear optics were first described in [17] although bistable hexagons and rolls have been investigated much earlier $[1,15]$. A typical example of such transverse structure is shown in Fig. 9. Note that nested patterns have high intensity values at the location of the fronts. In Fig. 10 we show that when decreasing $2 C$ from the honeycomb-roll structure the sequence of patterns ends with stable honeycombs at $2 C=18.6$, i.e. below the instability of the homogeneous solution at 18.8 . When increasing $2 C$ from coexisting patterns the honeycomb domain shrinks until wavy roll structures occur up to $2 C=19.95$ where stable rolls finally set in. An example of a typical wavy roll structure is shown in Fig. 11. An important and interesting point demonstrating again that the nature of the sequence of solutions depends on the chosen initial condition, is that the $2 C$-increasing scan of Fig. 10 does not end with the spontaneous formation of cavity solitons as observed for example in Figs. 5 and 6 . In scans with increasing values of $2 C$, the wavelength of the roll pattern is selected after the instability of the honeycomb or the growth from the unstable ho- 


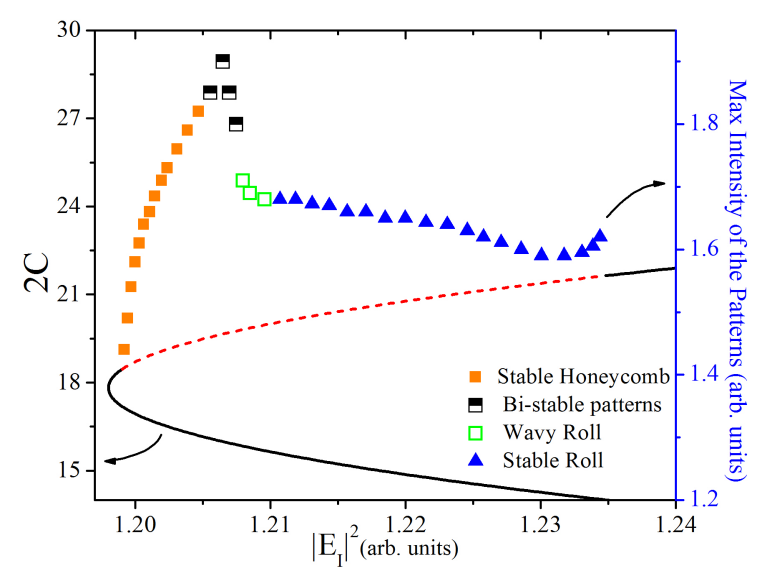

FIG. 10: (Color online) The sequence of patterns in input intensity versus $2 C$ space and the corresponding intensities for different patterns (right axis). These were obtained for an initial value of $2 C=19.60$.

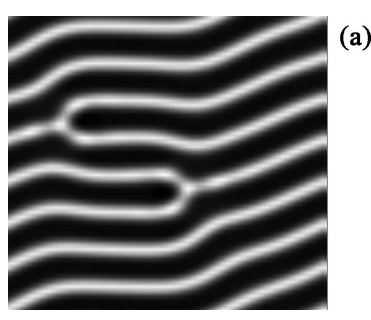

FIG. 11: Wavy roll pattern (a) and the corresponding far-field image (b) for $2 C=19.9$.

mogeneous state. This means that the roll patterns of Figs. 5, 6 and 10 have wave-vectors different from that of maximum growth. When approaching the end of the Turing unstable regions for large values of $2 C$, straight roll structures can encounter different instabilities that are wave-vector dependent such as Eckhaus, cross-roll, zig-zag and skewed-varicose instabilities [1, 4, 18]. Depending on the selected wave-vector of the roll structure of a particular scan, straight rolls may remain stable until the end of the Turing region (see Fig. 10) or lose their stability to, for example, gases of cavity solitons (see Figs. 5, 6) as discussed in the next subsection. Although there are similarities with what is observed in standard models such as Swift-Hohenberg [1, 4], Brusselator [1, 19] and Lugiato-Lefever [6], we note that in our case the stable homogeneous state for large $2 C$ is attained through a saddle-node bifurcation at $K=0$ instead of a Turing mechanism at finite wave-vector. We note that different instabilities for the same pattern with different wave-vector is not limited to the roll structures as discussed for example in [4] and in [20] for nonlinear optics.

The issue of different instability for the same pattern but different wave-vector is not limited to the roll structures discussed here and there are detailed studies that

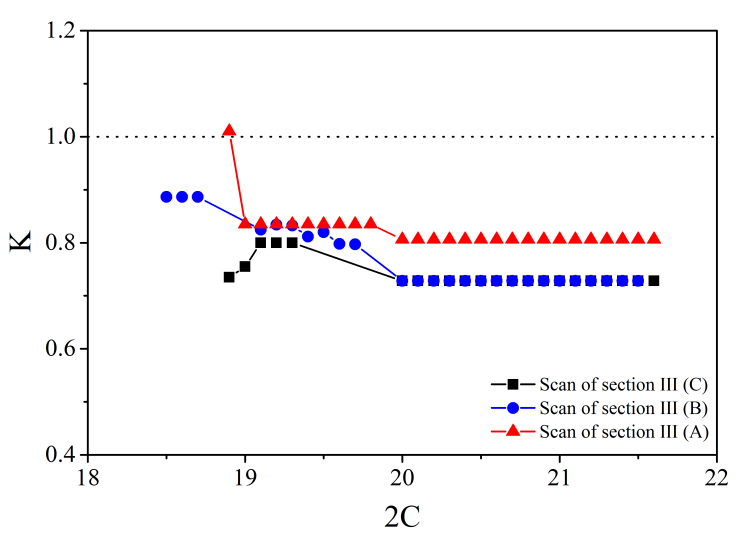

FIG. 12: (Color online) Deviations of the wave-vectors from that of maximum growth as a result of starting the scan at different distances from the threshold. Note that these values are for pure patterns, i.e. stable honeycombs forming before $2 \mathrm{C}=20$ and rolls thereafter. The missing parts in the curves are related to distorted honeycombs, bistable patterns and wavy rolls.

show such processes can happen for states that are periodic in two or more extended variables such as twodimensional lattice states, see for example section 4.2 and 4.3 of [4] for discussions on stability balloon.

The differences among the wave-vectors obtained in the three different scans presented above can be appreciated in Fig. 12. These curves confirm that the presence of pattern bistability and stable defects prevent the system to relax to the structure of maximum growth rate when changing a control parameter that simulates experimental realizations.

\section{Spontaneous formation of a gas of cavity solitons}

An intriguing feature of the transverse solutions displayed by EIT media in optical cavities is the spontaneous formation of a gas of cavity solitons for large values of the control parameter $2 C$ as observed in Figs. 5 and 6 . When following adiabatically the branch of the roll solutions, sudden instabilities perpendicular to the stripe structure occur. These instabilities are referred to as cross-roll instabilities and lead, in general, to the stabilisation of roll patterns perpendicular to and with a larger wave-vector than the original structure [18]. In our case, large wave-vector rolls are unstable and one observes a transient formation of distorted hexagons (sometimes rhomboids). Distorted hexagonal periodic structures are unstable in this regime. The low intensity homogeneous state is instead stable leading to the spontaneous formation of intensity peaks that weakly interact with each other in a gas of cavity solitons. The instability mechanism is illustrated in Fig. 13 where the roll pattern is shown to lose stability to a distorted hexagonal struc- 


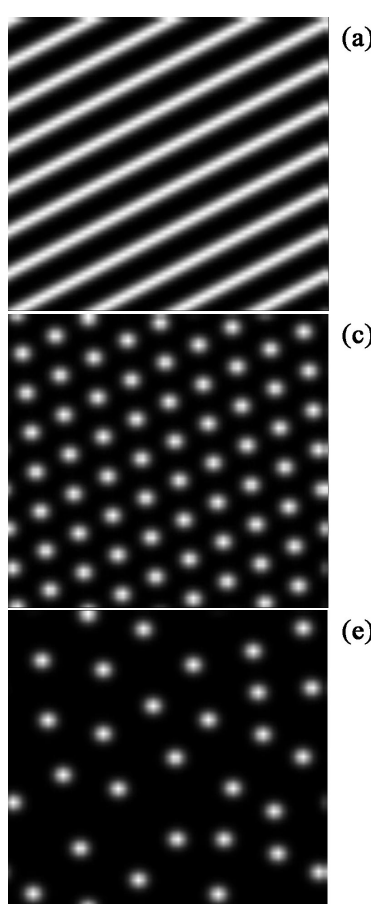

(a)

(b)
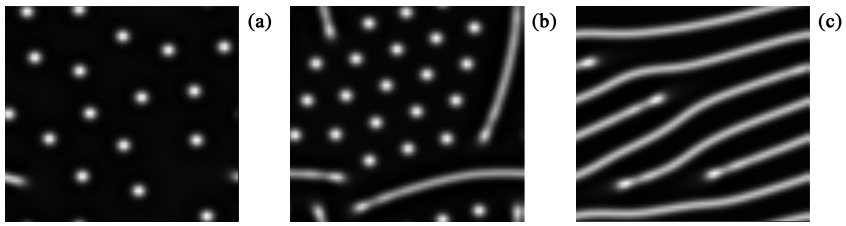

FIG. 14: Formation of filaments from stable cavity soliton gas by decreasing the control parameter. $2 C=20.88$ (a), $2 C=20.69(\mathrm{~b})$ and $2 C=20.65$ (c).

almost periodic structures made of filaments. Note that filament structures are bistable with regular rolls and further increase the number of multistable states of the EIT system.

Finally, in Fig. 15 we show the great variety of possible transverse structures that can be observed when changing the control parameter $2 C$ and the selected initial condition in an absorber close to EIT in a cavity. It is evident that the effect of initial condition includes the range of stability of pattern solutions, the presence or absence of stable defects, the appearance of special structures like wavy rolls, filaments or spontaneous cavity solitons, the onset of scrolling patterns and the overlap of two distinct pattern solutions. We note that this rich variety of structures and behaviour is not restricted to optics but is a universal feature of spatio-temporal nonlinear systems and has been observed for example in models for the calcification of the heart tissue [22].

\section{FINITE SIZE INPUT PUMP}

To understand the physical relevance in optics of the multistable solutions described in the previous section, it is important to investigate the effect of periodic boundary conditions used in the simulations. This can be properly done by comparing the previous results with those obtained in simulations with an input pump $P_{I}(r)$ of radial shape and finite size. This makes the situation closer to possible experimental realizations. In order to maintain a large aspect ratio in the transverse plane we have used a hyperbolic tangent profile which simulates a flat-top injected beam with rapidly vanishing tails:

$$
P_{I}(r)=\frac{E_{I}}{2}\left\{1-\tanh \left[\sigma\left(r-r_{0}\right)\right]\right\}
$$

where $\sigma$ and $r_{0}$ regulates the size of the tail and flat part of the pump, respectively. $P_{I}(r)$ replaces $E_{I}$ in Eq. (1). A grid of $128 \times 128$ points and size of $20 \lambda_{c}$ is used although checks on a $256 \times 256$ grid have been performed too.

All features described in Section III have been replicated in the simulations with finite size pumps apart from the scrolling structures with defects. Of course, the location of bifurcations and the nature of the asymptotic solution changes with the values of $\sigma$ and $r_{0}$, i.e. the size and shape of the input pump but the periodic, aperiodic 


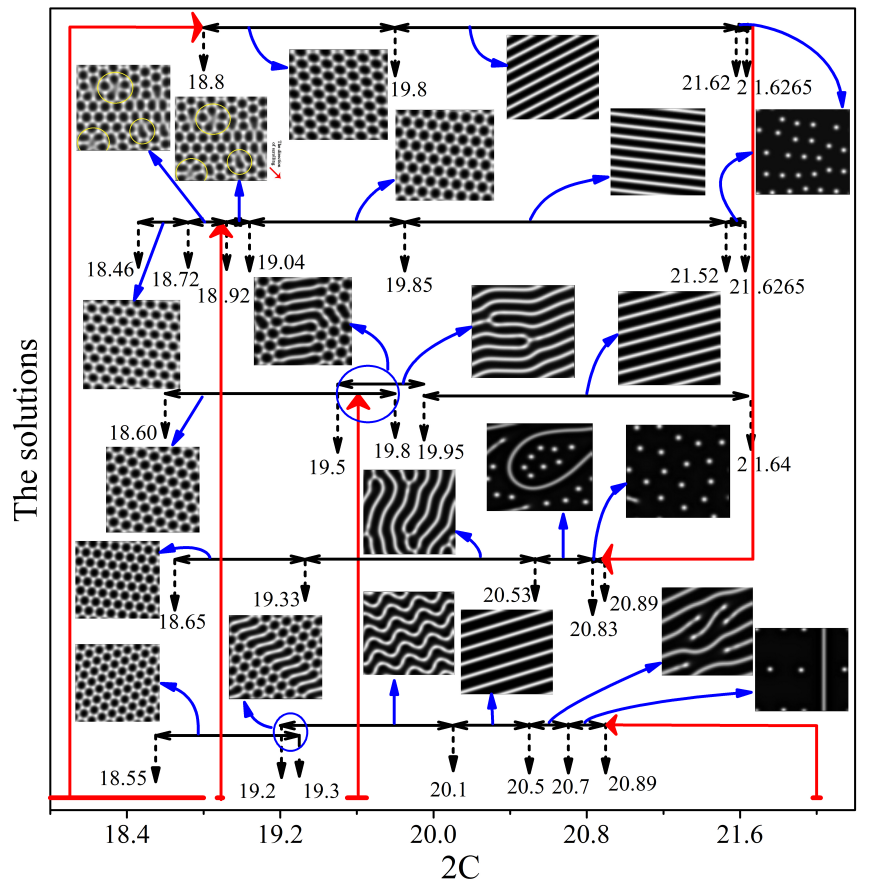

FIG. 15: Different progression of solutions as a consequence of selecting different initial conditions. The structures are stable honeycomb, stable roll, cavity solitons, distorted honeycomb, scrolling honeycombs, coexisting roll-honeycomb and wavy rolls respectively. Dashed arrows show the threshold for the patterns of the type which solid and longer arrows are pointed at. The sequence of scan in $2 \mathrm{C}$ for each set is shown by the arrows connecting the initial value, either from noise (points on the horizontal axis) or by using the memory of the last solution of a branch, to the set of solutions in branches. Note that cavity solitons are in a regime where the homogeneous background is stable, as seen from the right lower most sequence of solutions which starts from noise and shows the stability of homogeneous branch up to $2 \mathrm{C}=20.89$ where a saddle-node bifurcation occurs.

and localized structures displayed in Fig. 15 have found a counterpart in the simulations with circular injection. For example Fig. 16 shows patterns with defects, fronts between rolls and honeycombs (see [15] for a roll-hexagon structure with circular boundaries), filaments and cavity soliton gases respectively.

Of particular importance for our investigation is the robustness of the coexistence of rolls and honeycomb patterns. Fig. 16 (b) shows an example of stable roll patterns coexisting with honeycomb structures in the presence of a finite size injected pump. The introduction of two new parameters in the pump specification puts further emphasis on the crucial role of initial conditions in determining the final solutions and their sequence from the simple Eq. (1) because of generalised multistability of structures.

Differing from other multistable structures, the spontaneous scrolling motion of distorted patterns disappears as soon as one employs a finite pump size. The defects
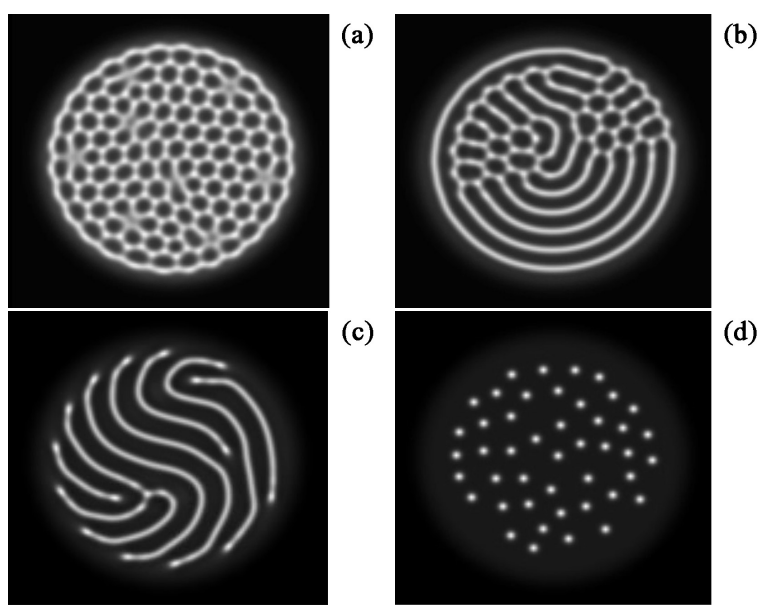

FIG. 16: Examples of transverse structures with a finite pump size with $\sigma=1.67 / \lambda_{c}$ and $r_{0}=9 \lambda_{c}$. Honeycomb with defects $(2 C=18.95)(a)$, coexistent honeycomb and roll structures $(2 C=19.6)(b)$, filaments $(2 C=20.50)(c)$ and gas of cavity solitons $(2 C=21.55)(\mathrm{d})$.

which are intrinsic to scrolling strictures [16] are instead a robust feature that survives the external imposition of circular symmetry as demonstrated in Fig. 16 (a).

\section{CONCLUSION}

Complex and multistable spatial structures forming in the self-focusing regime of a cavity close to EIT are discussed. The multistability of the model is explored by putting emphasis on the importance of initial conditions. Particularly, three routes of solutions are studied by numerical simulations proving unique properties for each. It is seen that the selection of initial value for the control parameter can affect the range of pattern solutions, presence or absence of specific patterns and their behaviours. We have also investigated nested patterns formed of stable rolls and honeycomb structures separated by stable fronts. Wave vector dependant roll instability is the mechanism responsible for the spontaneous formation of a gas of cavity solitons. Finally, the homogeneous pump has been replaced with a finite size injection to remove the effect of periodic boundary condition and check the robustness of the multistable solutions. All survived, showing their physical relevance in experimental conditions, with the exception of distorted scrolling patterns.

The multistability and rich variety of solutions are not a consequence of the specific selection of the parameters that have been kept at fixed values, i.e. $\Delta, \theta,\left|E_{2}\right|^{2}$ and $\left|E_{s}\right|^{2}$. We expect transverse structure multistability and dependence of the scans upon initial conditions over wide ranges of these parameter variations too. In particular we note that $\Delta$ controls the ratio of absorption versus dispersion in the EIT system. Its variation can be critical 
in the nature and bifurcations of the spatial structures observed. The generalized multistability observed here is a consequence of the feedback provided by the cavity mirrors and is expected to affect the output of the system even when medium propagation effects are taken into consideration. Propagation of light in media displaying EIT without a cavity leads to manipulation of the susceptibility in momentum space, slow light and even elimination of diffraction [23]. Coupling these features with our transverse cavity effects may offer a flexibility of operation that is unprecedented in nonlinear optical devices.

Finally, the onset and interaction of localized solutions such as cavity solitons and filaments in the EIT model appears to be different from what is observed in typical optical pattern formation with third order and second order nonlinearities. The transition from cavity solitons to filaments, the long range interaction of the solitons and the processes of control and use of localised solutions in photonic devices that display EIT will be the subject of future communications.

\section{Acknowledgements}

DM acknowledges EPSRC for support.
[1] D. Walgraef, Spatio-Temporal Pattern Formation, (Springer-Verlag, Berlin, 1997)

[2] L. A. Lugiato, M. Brambilla, and A. Gatti, Adv. At. Mol. Opt. Phys. 40, 229 (1999)

[3] T. Ackemann and W. J. Firth, in Dissipative Solitons edited by N. Akhmediev and A. Ankiewicz, Lecture Notes in Physics, 661 (Springer-Verlag, Berlin, 2006) page 55

[4] M. C. Cross and H. S. Greenside, Pattern Formation and Dynamics in Nonequilibrium Systems (Cambridge University Press, Cambridge, 2009)

[5] T. Ackemann, W. Firth, and G.-L. Oppo, Adv. At. Mol. Opt. Phys. 57, 323 (2009)

[6] L. A. Lugiato and R. Lefever, Phys. Rev. Lett. 58, 2209 (1987)

[7] J. Guckenheimer and P. Holmes, Nonlinear Oscillations, Dynamical Systems and Bifurcations of Vector Fields, (Springer-Verlag, Berlin, 1983)

[8] S. H. Strogatz, Nonlinear Dynamics and Chaos (Perseus, Cambridge, Massachusetts, 1994)

[9] D. Gomila and P. Colet, Phys. Rev. E 66, 046223 (2002); Phys. Rev. A 68, 011801R (2003).

[10] G.-L. Oppo, Journal of Modern Optics, 57, 1408 (2010).

[11] A. Joshi, A. Brown, H. Wang, and M. Xiao, Phys. Rev. A 67, 041801(R) (2003).

[12] L. A. Lugiato and C. Oldano, Phys. Rev. A 37, 3896
(1988).

[13] A. M. Turing, Phil. Trans. Royal Soc. London B 237, 37 (1952).

[14] G.-L. Oppo, J. Math. Chem. 45, 95 (2009).

[15] H. Xi, J. D. Gunton, and J. Vinals, Phys. Rev. E 47, R2987 (1993).

[16] A. J. Scroggie, D. Gomila, W. J. Firth, and G.-L. Oppo, App. Phys. B 81, 963 (2005).

[17] D. Michaelis, U. Peschel, and F. Lederer, Phys. Rev. A 56, 3366(R) (1997).

[18] P. Manneville, Dissipative Structures and Weak Turbulence, (Academic Press, London, 1990).

[19] A. De Wit, Advances Chem. Phys. 109, 435 (1999).

[20] G. K. Harkness, W. J. Firth, G.-L. Oppo and J. M. McSloy, Phys. Rev. E 66, 046605 (2002).

[21] C. McIntyre, A. M. Yao, G.-L. Oppo, F. Prati, and G. Tissoni, Phys. Rev. A 81, 013838 (2010).

[22] A. Yochelis, Y. Tintut, L. L. Demer, and A. Garfinkel, New J. of Phys. 10, 055002 (2008).

[23] O. Firstenberg, M. Shuker, N. Davidson, and A. Ron, Phys. Rev. Lett. 102, 043601 (2009); O. Firstenberg, P. London, M. Shuker, A. Ron, and N. Davidson, Nature Physics 5, 665 (2009). 